\title{
Delayed diagnosis of Townes-Brocks syndrome with multicystic kidneys and renal failure caused by a novel SALL1 nonsense mutation: A case report
}

\author{
FU-JUN LIN ${ }^{1 *}$, WEI LU $^{1 *}$, DANIEL GALE ${ }^{2}$, YAO YAO ${ }^{1}$, REN ZOU $^{3}$, FAN BIAN $^{1}$ and GENG-RU JIANG ${ }^{1}$ \\ ${ }^{1}$ Department of Nephrology, XinHua Hospital, School of Medicine, Shanghai Jiao Tong University, Shanghai 200092, \\ P.R. China; ${ }^{2}$ UCL Centre for Nephrology, Royal Free Campus, UCL Medical School, University College London, \\ London NW3 2PF, United Kingdom; ${ }^{3}$ Department of Medical Ultrasound, XinHua Hospital, \\ School of Medicine, Shanghai Jiao Tong University, Shanghai 200092, P.R. China
}

Received July 19, 2015; Accepted January 15, 2016

DOI: 10.3892/etm.2016.3035

\begin{abstract}
Townes-Brocks syndrome (TBS) is a rare autosomal dominant congenital anomaly syndrome characterized by the triad of anorectal, hand and external ear malformations. Kidney involvement is less common and may progress to end-stage renal failure (ESRF) early in life. The present study reports the case of a male patient presenting with multiple bilateral cortical kidney cysts at the age of 4 years, at which time the kidneys were of normal size and function. A clinical diagnosis of autosomal recessive polycystic kidney disease was made initially as the patient's parents are clinically healthy. However, the consideration of extra-renal involvements (imperforate anus at birth, preaxial polydactyly and dysplastic right ear) following the progression of the patient to ESRF at the age of 16 years, led to the diagnosis of TBS. This prompted sequencing of the SALL1 gene, which identified a novel heterozygous nonsense mutation in the mutational 'hotspot' of exon 2 (c.874C>T, p.Q292X), and this mutation was not detected in healthy controls. The current case highlights that TBS may present with normal sized, cystic kidneys in childhood, while recognition of extra-renal features of cystic kidney diseases, such as TBS, and genetic testing may facilitate the correct diagnosis and transmission mode. Reaching a
\end{abstract}

Correspondence to: Dr Geng-Ru Jiang, Department of Nephrology, XinHua Hospital, School of Medicine, Shanghai Jiao Tong University, 1665 Kong Jiang Road, Shanghai 200092, P.R. China

E-mail: jianggeng-ru@hotmail.com

Dr Daniel Gale, UCL Centre for Nephrology, Royal Free Campus, UCL Medical School, University College London, Rowland Hill Street, London NW3 2PF, United Kingdom

E-mail:d.gale@ucl.ac.uk

${ }^{*}$ Contributed equally

Key words: Townes-Brocks syndrome, multicystic kidneys, renal failure, $S A L L 1$, novel mutation correct diagnosis of as TBS is important since this condition has a $50 \%$ rate of transmission to offspring and can progress to ESRF early in life.

\section{Introduction}

Multiple renal cysts present clinical features in a range of kidney diseases that may be inherited as dominant or recessive traits (1). Autosomal dominant diseases include autosomal dominant polycystic kidney disease (ADPKD), which is by far the most common inherited cause of kidney cysts worldwide, as well as $H N F 1 B$-associated kidney disease, medullary cystic kidney disease (MCKD) and cystic neoplasms (such as Von Hippel-Lindau disease and tuberous sclerosis complex) (2). Although these conditions may present in children, they often cause adult-onset kidney disease, and a family history of kidney problems is frequently reported (3). Recessive conditions commonly present in childhood and often occur in the absence of a relevant family history. They include autosomal recessive polycystic kidney disease (ARPKD), nephronophthisis (NPHP) and multi-system ciliopathies, such as Bardet-Biedl, Joubert and Meckel-Gruber syndromes (4).

A range of other uncommon disorders characteristically presents with congenital abnormalities of the kidney and urinary tract (CAKUT), but may also manifest as cystic kidney disease (5). These diseases include: Townes-Brocks syndrome (TBS), which is caused by mutations in SALL1; renal coloboma syndrome, which is associated with $P A X 2$ mutations; oral facial digital syndrome type 1 , which is due to mutations in gene $O F D 1$; and other disorders that frequently include extra-renal manifestations (6). Imaging features of these disorders are variable and may overlap, while renal histology is frequently unavailable or does not help the diagnosis (7). Thus, recognition of extra-renal features is of critical importance in establishing the correct diagnosis. This is particularly valuable in cases where no family history is available, and therefore the differential diagnosis includes both dominant and recessive conditions. The mode of transmission determines whether there is a risk that other family members may be affected, thus affecting reproductive decision-making. Confirmation of the 
A

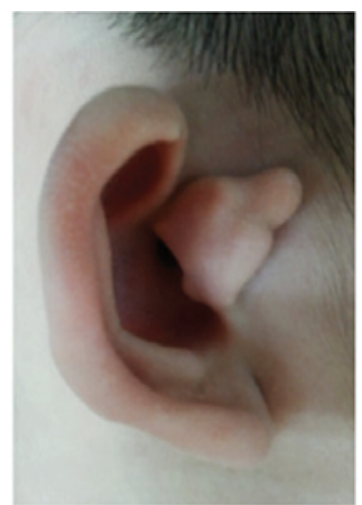

B

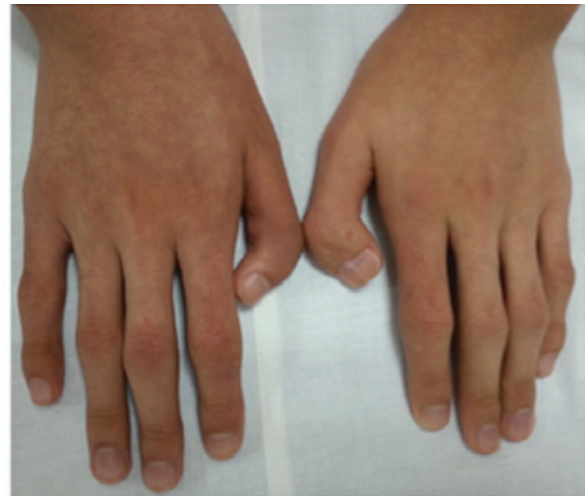

C
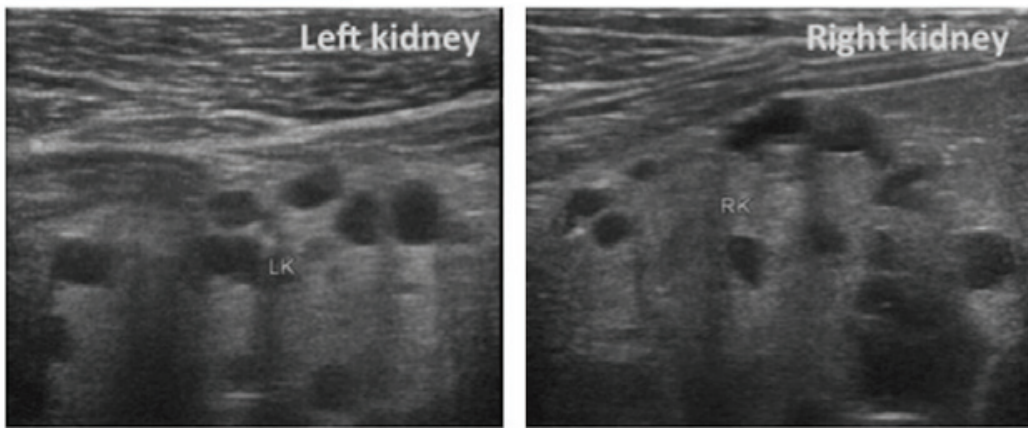

Figure 1. (A) Right ear of the proband with overfolded superior helices. (B) Surgically corrected thumbs of the proband's hands. (C) Renal ultrasound showed multiple cysts arising from the cortex and medulla of the left and right kidneys.

diagnosis by genetic testing can therefore be clinically valuable in this context.

The current study presents the case of a 16-year-old male patient with autosomal dominant TBS, who was initially incorrectly diagnosed as having ARPKD. Appreciation of extra-renal features prompted genetic testing that confirmed the correct diagnosis of an autosomal dominant disease, which has significant implications for the patient and family members.

\section{Case report}

The proband was a 16-year-old male who was referred to XinHua Hospital (Shanghai, China) in May 2014 with end-stage renal failure (ESRF) and a serum creatinine (Scr) level of $932 \mu \mathrm{mol} / 1$ (normal range, 35-97 $\mu \mathrm{mol} / \mathrm{l}$ ). The patient had been found to have proteinuria of 30-300 $\mathrm{mg} / \mathrm{dl}$ (normal range $0-20 \mathrm{mg} / \mathrm{dl}$ )) with no hematuria following an upper respiratory infection at the age of 4 years, in April 2002. There was no family history of kidney disease and his clinically healthy parents were not consanguineous. Ultrasound examination at the age of 4 years demonstrated that the patient's kidneys were a normal size for his age [left kidney, 69x33 mm; right kidney, $58 \times 31 \mathrm{~mm}$; reported normal kidney lengths for a 4-year-old, $70.30 \pm 5.20 \mathrm{~mm}(8)]$ with multiple bilateral cortical cysts (largest cyst, 10x17 mm). The Scr level $(45 \mu \mathrm{mol} / \mathrm{l})$ and blood pressure were within the normal range at that time. Liver function tests and hepatic imaging were also normal. The lack of family history and childhood onset of disease suggested a diagnosis of ARPKD, while ADPKD was also considered possible by the treating clinicians. The renal function gradually deteriorated and the Scr reached a level of $400 \mu \mathrm{mol} / 1$ at the age of 15 years, with renal dialysis commencing at the age of 16 years at the Dialysis Center of XinHua Hospital.
Upon reviewing the medical history, it was found that the patient had an imperforate anus at birth, which was surgically repaired when he was 3 months old, as well as pre-axial polydactyly of both hands, which was surgically corrected when the patient was 3 years old. The patient was also reported to have exhibited mild bilateral hearing loss since the age of 5 years. However, no growth retardation or learning difficulties were reported. Physical examination revealed a dysplastic right ear with overfolded superior helices (Fig. 1A), as well as bilateral surgically corrected thumbs (Fig. 1B). Renal ultrasound examination performed on an Ultrasound LOGIQ 500 (GE Healthcare Milwaukee, WI, USA) showed bilateral small kidneys (left kidney, 74x36 mm; right kidney, $59 \times 31 \mathrm{~mm}$ ) with multiple cortical and medullary cysts, ranging between 10 and $17 \mathrm{~mm}$ (Fig. 1C). No other urinary tract structural abnormality was identified, imaging assessments did not detect any abnormalities of the liver, spleen or pancreas, and an echocardiogram was normal. The parents were clinically unaffected and no family history of genetic disease was reported.

Based on the aforementioned extra-renal features, a diagnosis of TBS was suspected. Following approval by the Ethical Committee of Shanghai XinHua Hospital according to the standards of the Declaration of Helsinki and the signed informed consent obtained from the proband, DNA was extracted from the proband's peripheral blood. SALL1 gene coding regions and splice sites were amplified and directly sequenced using an ABI PRISM 3730xl Genetic Analyzer (Applied Biosystems; Thermo Fisher Scientific, Inc., Waltham, MA, USA) as reported previously (9), (SALLI sequencing primer sequences are available upon request). Sequencing analysis disclosed a novel SALL1 heterozygous nonsense mutation located in the mutational 'hotspot' of 
A
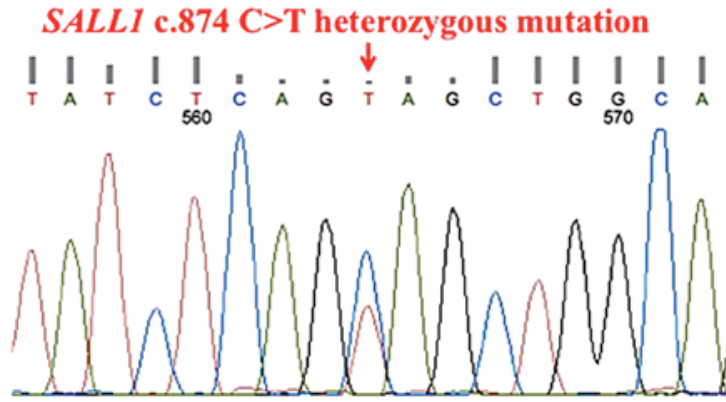

B

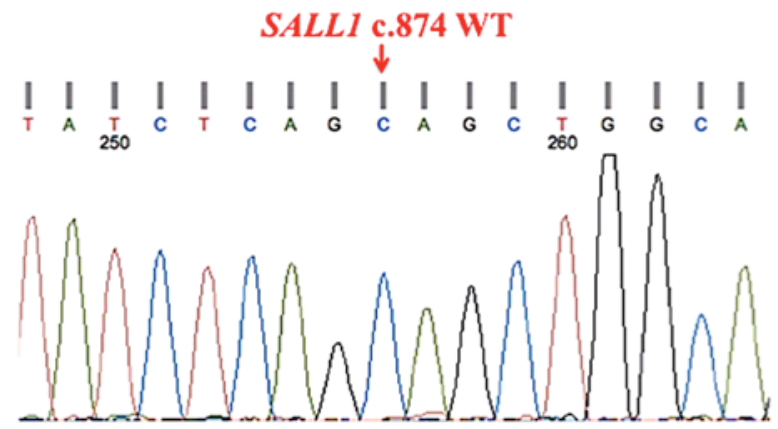

Figure 2. Sanger sequencing electropherograms showed that (A) the proband has the SALL1 heterozygous mutation (c.874C>T, p.Q292X), (B) which was not detected in 100 healthy Chinese controls.

exon 2 (c.874C>T, p.Q292X; Fig. 2A), and this mutation was not present in the 100 unrelated healthy adult volunteers (all of whom provided informed consent, were free from renal disease and matched geographically) used as controls (Fig. 2B). The parents of the proband declined genetic testing, however it appears likely that this represents a de novo mutation, which is estimated to occur in $~ 50 \%$ of patients with TBS (10), as the parents of the proband are clinically healthy. It is also possible that one of the parents carries the mutation in a mosaic state, as germline mosaicism in clinically unaffected parents has been reported in TBS cases (11). As the patient had reached ESRF at the time of genetic diagnosis, the patient remained on maintenance hemodialysis three times per week until the final follow-up in March 2015.

\section{Discussion}

TBS has a prevalence of 1 in 250,000 individuals in the general population (12). It is a rare autosomal dominant malformation syndrome characterized by the core triad of anorectal malformation (imperforate anus, anteriorly-placed anus and anal stenosis), hand malformations (pre-axial polydactyly, triphalangeal thumbs and bifid thumb) and external ear malformation (preauricular ear tags and overfolding of ear helices) associated with deafness (13). TBS is confirmed by identification of a pathogenic mutation in SALL1 with the detection rate of $64-83 \%$ (14). SALL1 is a human gene homologous to the developmental regulator sal of Drosophila melanogaster, which is considered to be an essential organogenesis regulator for urological, renal, limb, ear, brain and liver development (15).

Sall1 protein, encoded by $S A L L 1$, is abundantly expressed in embryonic kidneys and plays a pivotal role in mammalian nephrogenesis by controlling the expression of major renal development genes, including PAX8, GDNF and FOXDI (16). Mice with homozygous deletion of Sall1 $\left(S A L L 1^{--}\right)$have been observed to succumb to death in the perinatal period due to renal agenesis or severe dysgenesis (17), while another mouse model expressing a truncated N-terminal protein (Sall1-N) recapitulated human TBS renal defects of renal hypoplasia with or without cysts (18). The incidence of renal anomalies in TBS patients ranges between 20 and $62.5 \%$, with heterogeneous phenotypes including unilateral or bilateral renal hypoplasia or dysplasia, renal agenesis, multicystic kidneys, vesicoureteral reflux, posterior urethral valve or meatal stenosis (19). In addition, renal hypodysplasia has also been reported as an isolated TBS phenotype (20). A study involving 749 patients with CAKUT revealed that SALL1 mutations account for $>20 \%$ of all the identified mutations ( 9 out of 37 mutations), indicating that this mutation is more common than was expected (21). Therefore, kidney imaging is recommended for TBS patients and in case presenting renal anomalies. Furthermore, close monitoring of renal function and appropriate medical interventions are indicated, as TBS has been reported to be associated with early-onset renal failure with nearly $15 \%$ of TBS patients with renal anomalies progressing to ESRF between the ages of 1 and 23 years (19).

The patient reported in the present study harbored a novel heterozygous nonsense mutation (c.874C >T, p.Q292X) in the SALL1 mutational hotspot region. This mutational hotspot region is located within exon 2 between nucleotide $765,3^{\prime}$ of nucleotides 687-750 encoding the glutamine-rich interaction domain, and nucleotide $1565,3^{\prime}$ of the region encoding the first double zinc finger domain (22). Frameshift and nonsense SALL1 mutations occur in this mutational hotspot region, such as the most common SALL1 heterozygous mutation, which is c.826C $>$ T, p.R276X. These mutations are usually associated with classical TBS and a more severe phenotype, including renal failure $(10,19,22)$, which suggests a dominant-negative or gain-of-function mechanism caused by the abnormal truncated Sall1 protein. In comparison, haploinsufficiency for SALL1 caused by heterozygous large or whole gene deletions have been described in milder TBS cases (9). Only one previous study has described renal insufficiency and ESRF in two Japanese adolescent patients with 16q12 microdeletion including the entire SALL1 gene (23).

In the present TBS case, the correct diagnosis was delayed until the patient reached ESRF, suggesting that recognition of extra-renal features is of critical importance in making a diagnosis in cystic kidney diseases, particularly when renal imaging is atypical. The possibility that clinical features of TBS may overlap with other rare developmental syndromes such as VACTERL association (24), Goldenhar syndrome (25) and branchiootorenal (BOR) syndrome (26), should also be considered. Additional features, such as vertebral abnormalities and trachea-esophageal fistula in VACTERAL association, craniofacial abnormalities in Goldenhar syndrome and branchial arch anomalies in BOR syndrome, may aid the diagnosis of these disorders.

In conclusion, the present case illustrated that TBS may present with normal-sized cystic kidneys in childhood. Recognition of extra-renal features of cystic kidney diseases, such as TBS, and genetic testing may facilitate the correct 
diagnosis, which is necessary to give the correct advice to patients and their families regarding the mode of transmission and consequent risk to relatives and offsprings of those affected. As TBS can cause ESRF early in life, renal imaging and close monitoring of renal function are recommended for TBS patients with renal anomalies.

\section{Acknowledgements}

The present study was supported by the National Natural Science Foundation of China (grant no. 81500507), the Young Investigator Funding provided by the Shanghai Health Bureau (grant no. 20114Y108) and the SMC-Young Investigator Funding (grant no. 2013SMCYIF01) provided by Shanghai Jiao Tong University.

\section{References}

1. Bergmann C: ARPKD and early manifestations of ADPKD: The original polycystic kidney disease and phenocopies. Pediatr Nephrol 30: 15-30, 2015.

2. Ong AC, Devuyst O, Knebelmann B and Walz G; ERA-EDTA Working Group for Inherited Kidney Diseases: Autosomal dominant polycystic kidney disease: The changing face of clinical management. Lancet 385: 1993-2002, 2015.

3. Schrier RW, Brosnahan G, Cadnapaphornchai MA, Chonchol M, Friend K, Gitomer B and Rossetti S: Predictors of autosomal dominant polycystic kidney disease progression. J Am Soc Nephrol 25: 2399-2418, 2014.

4. Hoyer PF: Clinical manifestations of autosomal recessive polycystic kidney disease. Curr Opin Pediatr 27: 186-192, 2015.

5. Devuyst O, Knoers NV, Remuzzi G and Schaefer F: Board of the Working Group for Inherited Kidney Diseases of the European Renal Association and European Dialysis and Transplant Association: Rare inherited kidney diseases: Challenges, opportunities, and perspectives. Lancet 383: 1844-1859, 2014.

6. Hwang DY, Dworschak GC, Kohl S, Saisawat P, Vivante A, Hilger AC, Reutter HM, Soliman NA, Bogdanovic R, Kehinde EO, et al: Mutations in 12 known dominant disease-causing genes clarify many congenital anomalies of the kidney and urinary tract. Kidney Int 85:1429-1433,2014.

7. Kiser RL, Wolf MT, Martin JL, Zalewski I, Attanasio M, Hildebrandt F and Klemmer P: Medullary cystic kidney disease type 1 in a large Native-American kindred. Am J Kidney Dis 44: 611-617, 2004

8. Luk WH, Lo AX, Au-Yeung AW, Liu KK, Woo YH, Chiang CC and Lo KK: Renal length nomogram in Hong Kong Asian children: Sonographic measurement and multivariable approach J Paediatr Child Health 46: 310-315, 2010.

9. Miller EM, Hopkin R, Bao L and Ware SM: Implications for genotype-phenotype predictions in Townes-Brocks syndrome: Case report of a novel SALL1 deletion and review of the literature. Am J Med Genet A 158A: 533-540, 2012.

10. Reardon W, Casserly LF, Birkenhäger R and Kohlhase J: Kidney failure in Townes-Brocks syndrome: An under recognized phenomenon? Am J Med Genet A 143A: 2588-2591, 2007.
11. Kohlhase J, Taschner PE, Burfeind P, Pasche B, Newman B, Blanck C, Breuning MH, ten Kate LP, Maaswinkel-Mooy P, Mitulla B, et al: Molecular analysis of SALL1 mutations in Townes-Brocks syndrome. Am J Hum Genet 64: 435-445, 1999.

12. Eker HK, Balasar Ö: Variable expressivity of renal involvement in a further family with Townes-Brocks syndrome. Clin Dysmorphol 24: 24-25, 2015.

13. Lawrence C, Hong-McAtee I, Hall B, Hartsfield J, Rutherford A Bonilla $\mathrm{T}$ and Bay C: Endocrine abnormalities in Townes-Brocks syndrome. Am J Med Genet A 161A: 2266-2273, 2013.

14. Albrecht B, Liebers M and Kohlhase J: Atypical phenotype and intrafamilial variability associated with a novel SALL1 mutation. Am J Med Genet A 125A: 102-104, 2014.

15. Kohlhase J, Wischermann A, Reichenbach H, Froster U and Engel W: Mutations in the SALL1 putative transcription factor gene cause Townes-Brocks syndrome. Nat Genet 18: 81-83, 1998.

16. Basta JM, Robbins L, Kiefer SM, Dorsett D and Rauchman M: Sall1 balances self-renewal and differentiation of renal progenitor cells. Development 141: 1047-1058, 2014.

17. Nishinakamura R, Matsumoto Y, Nakao K, Nakamura K, Sato A, Copeland NG, Gilbert DJ, Jenkins NA, Scully S, Lacey DL, et al: Murine homolog of SALL1 is essential for ureteric bud invasion in kidney development. Development 128: 3105-3115, 2001

18. Kiefer SM, Ohlemiller KK, Yang J, McDill BW, Kohlhase J and Rauchman M: Expression of a truncated Sall1 transcriptional repressor is responsible for Townes-Brocks syndrome birth defects. Hum Mol Genet 12: 2221-2227, 2003.

19. Faguer S, Pillet A, Chassaing N, Merhenberger M, Bernadet-Monrozies P, Guitard J and Chauveau D: Nephropathy in Townes-Brocks syndrome (SALL1 mutation): Imaging and pathological findings in adulthood. Nephrol Dial Transplant 24: 1341-1345, 2009.

20. Weber S, Moriniere V, Knüppel T, Charbit M, Dusek J, Ghiggeri GM, Jankauskiené A, Mir S, Montini G, Peco-Antic A, et al: Prevalence of mutations in renal developmental genes in children with renal hypodysplasia: Results of the ESCAPE study. J Am Soc Nephrol 17: 2864-2870, 2006.

21. Hwang DY, Dworschak GC, Kohl S, Saisawat P, Vivante A, Hilger AC, Reutter HM, Soliman NA, Bogdanovic R, Kehinde EO, et al: Mutations in 12 known dominant disease-causing genes clarify many congenital anomalies of the kidney and urinary tract. Kidney Int 85: 1429-1433, 2014.

22. Botzenhart EM, Bartalini G, Blair E, Brady AF, Elmslie F, Chong KL, Christy K, Torres-Martinez W, Danesino C, Deardorff MA, et al: Townes-Brocks syndrome: Twenty novel SALL1 mutations in sporadic and familial cases and refinement of the SALL1 hot spot region. Hum Mutat 28: 204-205, 2007.

23. Morisada N, Sekine T, Ishimori S, Tsuda M, Adachi M, Nozu K, Nakanishi K, Tanaka R and Iijima K: 16q12 microdeletion syndrome in two Japanese boys. Pediatr Int 56: e75-e78, 2014.

24. Solomon BD: VACTERL/VATER association. Orphanet J Rare Dis 6: 56, 2011.

25. Ashokan CS, Sreenivasan A and Saraswathy GK: Goldenhar syndrome-review with case series. J Clin Diagn Res 8: ZD17-ZD19, 2014

26. Engels S, Kohlhase J and McGaughran J: A SALL1 mutation causes a branchio-oto-renal syndrome-like phenotype. J Med Genet 37: 458-460, 2000. 